\title{
Stem Cell Fate Analysis Revisited: Interpretation of Individual Clone Dynamics in the Light of a New Paradigm of Stem Cell Organization
}

\author{
Ingo Roeder, Katrin Braesel, Ronny Lorenz, and Markus Loeffler \\ Institute for Medical Informatics, Statistics and Epidemiology (IMISE), University of Leipzig, 04107 Leipzig, Germany
}

Received 1 November 2006; Revised 2 January 2007; Accepted 21 January 2007

Recommended by James L. Sherley

\begin{abstract}
Many experimental findings on heterogeneity, flexibility, and plasticity of tissue stem cells are currently challenging stem cell concepts that assume a cell intrinsically predefined, unidirectional differentiation program. In contrast to these classical concepts, nonhierarchical self-organizing systems provide an elegant and comprehensive alternative to explain the experimental data. Here we present the application of such a self-organizing concept to quantitatively describe the hematopoietic stem cell system. Focusing on the analysis of individual-stem-cell fates and clonal dynamics, we particularly discuss implications of the theoretical results on the interpretation of experimental findings. We demonstrate that it is possible to understand hematopoietic stem cell organization without assumptions on unidirectional developmental hierarchies, preprogrammed asymmetric division events or other assumptions implying the existence of a predetermined stem cell entity. The proposed perspective, therefore, changes the general paradigm of thinking about stem cells.
\end{abstract}

Copyright (C) 2007 Ingo Roeder et al. This is an open access article distributed under the Creative Commons Attribution License, which permits unrestricted use, distribution, and reproduction in any medium, provided the original work is properly cited.

\section{INTRODUCTION}

Is this particular cell a stem cell? Any attempt to answer this question implies the idea that one can prospectively decide about the capabilities of a selected cell without relating it to other cells and without functionally testing its capabilities. This, however, might be a rather unrealistic point of view. To explain this, consider the definition of tissue stem cells. It is widely accepted that currently a definite characterization of tissue stem cells is only possible on the basis of their functional capabilities and not on the basis of explicit, directly observable attributes. Such a functional perspective is inherently consistent with the biological role of tissue stem cells to maintain tissue homeostasis and to (re)generate functional tissues.

The two key capabilities of tissue stem cells are the ability to self-renew their own population and the ability to produce a large number of fully functional, differentiated cells, implying also the ability to proliferate. However, although these are necessary capabilities, they are not sufficient to guarantee long-term maintenance and reconstitution of a fully functional tissue, which requires a highly coordinated control of cell production and differentiation. This points to another essential property of tissue stem cells: the flexibility in the use of their functional potentials. This flexibility, which had for the first time been incorporated into a definition of tissue stem cells by Potten and Loeffler [1], refers to the fact that stem cells might particularly be characterized by their ability to respond to the actual needs of the system. Such adaptiveness inevitably requires a communication of stem cells among each other and with their microenvironment. Beside feedback regulations on the basis of long-range acting molecules such as cytokines [2-4], this communication also refers to the importance of the so-called stem cell niche [59]. Meanwhile, the existence of stem cell supporting niches has been identified for most (regenerative) tissues, including the hematopoietic system $[10,11]$. Moreover, there is increasing evidence that stem cell organization is the result of complex cell-cell and cell-microenvironment interactions rather than the consequence of a predefined stem cell intrinsic program [12-15].

Applying the functional definition, the above-stated question whether a particular cell is a stem cell can only be answered retrospectively, having subjected the cell to a functional assay. This, however, will induce a cellular response and will inevitably alter the actual properties of the cell. This 
means that, in order to answer the question, one unavoidably loses the original cell. This situation is somehow similar to Heisenberg's uncertainty principle in quantum physics which states that the very act of measuring the functional properties of a certain system always changes its characteristics, thus, giving rise to a certain degree of uncertainty in the evaluation of the system properties. Although not identical, the uncertainty in the determination of the functional potential of a cell still implies that all prospective statements about stem cell functioning are necessarily probabilistic statements about the cellular behavior under particular conditions.

\section{CHALLENGES IN STEM CELL BIOLOGY}

There are a number of experimental observations which challenge the classical conception of a cell intrinsically predefined stem cell program. Although these observations are not restricted to one particular tissue, we will discuss them with the focus on the hematopoietic system.

Hematopoietic stem cells (HSCs) are heterogeneous with respect to functional properties such as cycling activity, engraftment potential or differentiation status, as well as to the expression of specific markers (phenotypic heterogeneity). Although there exist a number of sophisticated purification protocols that are able to select more homogeneous populations of stem cells [16-20], there is always a certain functional overlap of the obtained subpopulations. Furthermore, there is accumulating evidence that the phenotypic properties of HSC are reversibly changing (phenotypic reversibility) [21-28] and that tissue stem cells specified for one type of tissue can be manipulated such that they can act as stem cells of another tissue (stem cell plasticity) [29-32]. Even though there are most likely a number of constraints in the developmental options, these observations point to the fact that the functional potential of a stem cell cannot be uniquely determined by its actual phenotypic appearance. Therefore, although a specific purification protocol might select a population of cells with a homogeneous phenotype, showing a certain behavior within a particular functional assay, this behavior might change over time or if the cells are exposed to different assay conditions.

Because classical stem cell concepts are not able to explain all these experimental findings consistently, new conceptual approaches are required. However, to be validated, such concepts need a rigorous examination by quantitative and predictive modeling approaches.

\section{THEORETICAL CONCEPTS AND QUANTITATIVE MODELS IN STEM CELL BIOLOGY}

Particularly with respect to the uncertainty in the prospective characterization of stem cell function, a well-defined theoretical framework will help to cope with the complexity of experimental systems and will, therefore, considerably contribute to a deeper understanding of functional principles of stem cell organization. In conjunction with predictive quantitative models, such a theory will assist biologists to select, design, and optimize experimental strategies, and can help to systematically anticipate the impact of manipulations to a system. Theoretical approaches and simulation techniques support the identification of latent mechanisms and crucial parameters of biological processes, and may predict new phenomena. Furthermore, the application of a common model structure to different systems (i.e., tissues or cell types) may help to understand generic construction and regulation principles.

To serve as the basis for a theoretical framework of tissue stem cell organization and to allow for a stringent experimental validation of the theory, quantitative models have to fulfill a number of general requirements. They have to provide experimentally testable predictions. Because functional assays are the only way to definitely characterize tissue stem cells, the models must be able to account for the readouts of these assays. This requires that system-measurement interactions have to be considered in the model. Furthermore, stem cell models must be based on populations of individual cells to follow clonal development, to enable considerations of population fluctuations, and to conform to the uncertainty principle. Because of the increasing evidence that stem cell behavior is not the result of a cell-autonomous program, but instead the consequence of complex cell-cell and cell-growth environment interactions, these interactions have to be represented in such models. To be able to correctly describe regulatory processes, the model systems have to be dynamic in time, and possibly also in space. Particularly, they must be comprehensive in the sense of being applicable to normal homeostasis as well as to perturbed situations.

\section{A NEW PERSPECTIVE ON STEM CELL SYSTEMS}

The functional definition of tissue stem cells implies that stemness should be regarded as a functional endpoint rather than as an explicit attribute of individual cells. Therefore, any concept of tissue stem cells has to specify assumptions about the mechanisms that potentially control the regenerative and proliferative potential of these cells. Thus, a dynamic model should adequately represent processes that drive and control cellular attributes. Apparently, these processes are determined by the genetic and epigenetic statuses of the cells as well as by the activity of various signaling and metabolic pathways. Since it is presently impossible to describe the entirety of these processes in any reasonable detail, one major goal is the derivation of a simplified basic scheme accounting for the generic principles underlying the cellular dynamics.

Because many experimental results show the necessity to consider flexibility and reversibility of cellular properties as important constituents of stem cell organization, we propose to give up the view of tissue stem cells as being entities with a preprogrammed development. This view should be replaced by a concept that makes cellular capabilities for flexible and regulated tissue self-organizing the new paradigm [13]. Such a concept incorporates context-dependent phenotypic reversibility and generation of stem cell heterogeneity as the result of a dynamically regulated process. It consequentially avoids assumptions that lead to a direct or indirect $a$ priori labeling of particular cells as stem cells; cells are purely 
characterized on the basis of functional potentials. These cellular potentials as well as their actual use are able to change in response to cell-cell and cell-microenvironment interactions, such that the cell population fulfils the functional criteria of the stem cell definition. In this sense, a cell with high potential for long-term repopulation will not necessarily act as a long-term repopulating cell. In contrast, a cell with only a low long-term repopulating potential might, under certain circumstances, be selected to act as a stem cell. It should be stressed that although this concept includes a considerable degree of flexibility in the cellular development, it does not exclude the existence of restrictions in the developmental potential of individual cells. Therefore, also the complete loss of repopulating potential at a certain stage of development (e.g., due to terminal differentiation) is compatible with the proposed concept.

To put such a theoretical framework to a quantitative test, comparing it with various types of experimental observations, the general concept has been translated into a stochastic, single-cell-based model for HSC [33] which is summarized in the next section.

\section{A NEW MODEL OF HEMATOPOIETIC STEM CELL ORGANIZATION}

As already described in the context of the general concept, we assume that cellular properties of HSC can reversibly change within a range of potential options. Herein, the direction of cellular development and the decision whether a certain property is actually expressed depend on the internal state of the cell and on signals from its growth environment. Particularly, individual cells are considered to reside in one of two growth environments (denoted as GE-A and GE- $\Omega$ ). The state of each cell is characterized by its actual growth environment, by its position in the cell cycle $\left(G_{1}, S, G_{2}, M\right.$, or $\left.G_{0}\right)$, and by a property $a$, which describes its affinity to reside in GE-A. Whereas cells in GE- $\Omega$ are assumed to gradually loose affinity $a$, cells in GE-A are able to gradually regain $a$ (up to a maximal value $a_{\max }$ ). Furthermore, whereas cells in GE-A are assumed to be nonproliferating, cells in GE- $\Omega$ are able to proliferate with an average generation time $\tau_{c}$. The transition of cells between the two growth environments is modeled as a stochastic process. The transition intensities (i.e., the probabilities of growth environment change per time step, denoted as $\alpha$ and $\omega$ ) depend on the actual value of the affinity $a$ and on the number of stem cells residing in GE-A and GE- $\Omega$, respectively. If affinity $a$ of an individual cell has fallen below a prespecified threshold $\left(a_{\mathrm{min}}\right)$, the ability to home to GE-A and, therefore, the potential to regain affinity $a$ is lost. These cells start the formation of differentiated clones with a fixed life span, that is, they continue to proliferate for a fixed period of time and are finally removed from the system. Figure 1 provides a graphical illustration of the model structure.

We demonstrated that this model of HSC organization consistently describes a broad variety of observed phenomena such as heterogeneity of clonogenic and repopulation potentials, changing cell cycle activity of primitive progenitors, or different types of clonal competition including the

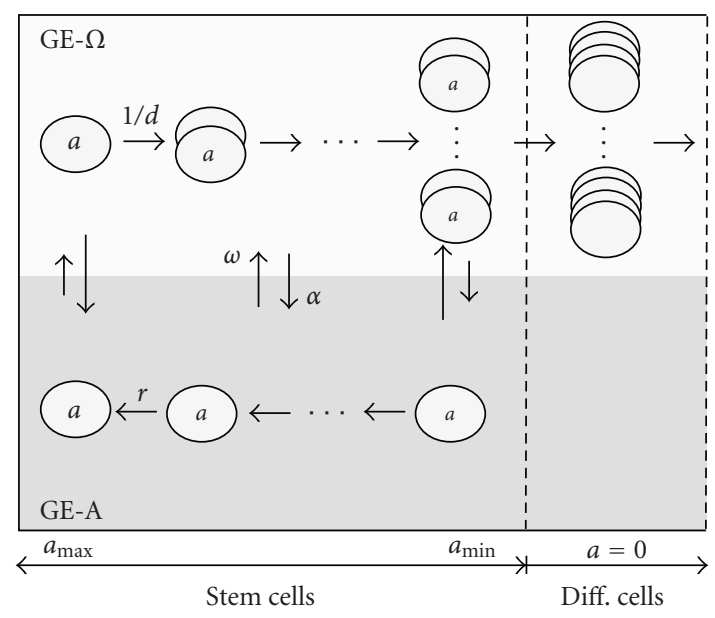

FIgURE 1: Schematic representation of the model. The lower part (gray) represents growth environment GE-A and the upper part (white) GE- $\Omega$. Cell amplification due to proliferation in GE- $\Omega$ is illustrated by growing cell numbers. Whereas growth environment affinity $a$ decreases by factor $1 / d$ per time step in GE- $\Omega$, it increases by factor $r$ per time step in GE-A. The actual quantity of $a$ is sketched by different font sizes. If $a$ falls below a critical threshold $a_{\text {min }}$, the cell loses its potential to switch to GE-A and $a$ is set to zero (represented by empty cells). These cells are called differentiated. Transition between GE-A and GE- $\Omega$ occurs with intensities $\alpha$ and $\omega$, which depend on $a$ (represented by the differently scaled vertical arrows) and on the cell numbers in the target GE (reprinted from [33] with permission from International Society for Experimental Hematology).

development and treatment of specific human leukemias [33-36]. Particularly, the proposed single-cell-based model structure allows to analyze cellular dynamics not only on the population, but also on the individual clone level. This is of particular interest in applications where the dynamic properties of individual (potentially manipulated) stem cells or stem cell clones are essential targets. Examples of such applications are gene-therapeutic approaches, and also the ex vivo expansion of stem or progenitor cells. In both cases, the competitive repopulation potential and the in vivo persistence of (clonally derived) stem cell transplants should be controlled and possibly optimized.

To illustrate the theoretical investigation of individual cell fates and of clonal dynamics and to highlight important benefits of a model analysis, we will consider two particular phenomena classes: fluctuating contribution of individually marked stem cell clones and cell fate asymmetry of paired progenitors.

\section{CLONALITY ANALYSIS ON THE SINGLE-CELL LEVEL}

To simulate the dynamics of individual stem cell clones, all model cells are individually labeled with an inheritable marker at one point in time. Using this procedure, it is possible to track all clones, initiated by these cells. We would like to unmistakably point out that here and throughout the paper, a clone is defined as the entire progeny of one particular cell. 


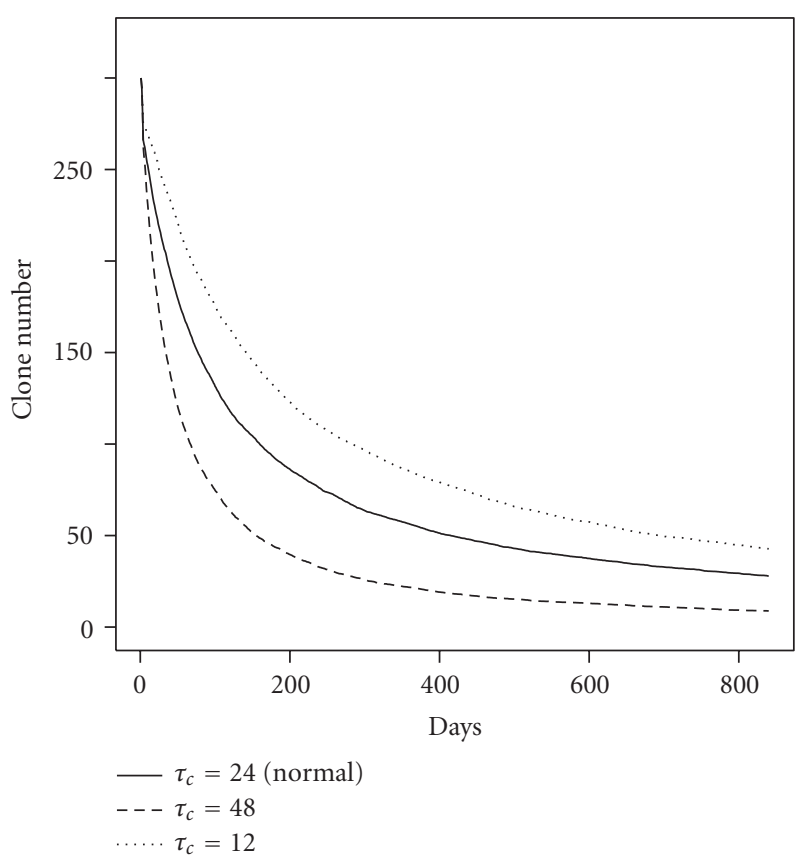

(a)

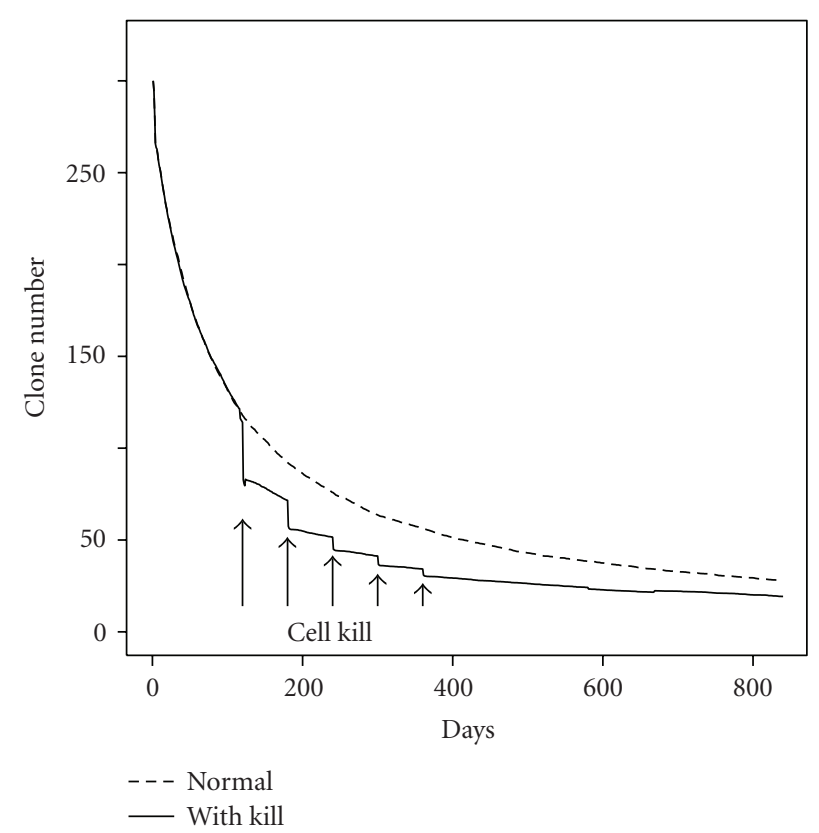

(b)

Figure 2: Clonality conversion. The numbers of existing clones within a homeostatic model system starting from an individual labeling of all stem cells at time zero (average of 20 simulation runs) is shown. Clonal conversion dependent on (a) average generation time $\tau_{c}$ (in hours) and on (b) repeated system disturbances (killing $50 \%$ of all stem cells at each indicated time point).

This implies that a clone is always characterized relative to a particular marking event, specifying the founder cell of the clone. It is also possible that different marking events define nested clones, implying that identical cells can be considered as members of different clones.

Consider the case that the individual cell marking procedure is completely neutral (i.e., not inducing any competitive growth advantage) and has been applied to a homeostatic hematopoietic system. This means that the number of traceable clones equals the total number of cells contributing to the system at this particular time point. Starting from such a configuration, our model predicts that the system will inevitably convert from this polyclonal state to an oligo- and finally to a monoclonal situation. In other words, asymptotically all cells will belong to only one clone (i.e., all having one common ancestor) even in the case of completely neutral marking. However, the time scale of such a monoclonality conversion might be very large. For the murine homeostatic reference situation (see [35] for detailed model parameters) with about 300 model stem cells, the time to monoclonality has been estimated to be approximately 65 years. During a normal mouse life span of about 2 years, the number of stem cell clones is predicted to reduce to about 30 . The cause of this clonality conversion is the stochastic fluctuation of cells between the two growth environments, with a certain positive probability of final differentiation (here, in the sense of reaching $a<a_{\min }$ ) for cells in GE- $\Omega$. Of course, the kinetics of the conversion depends on the model parameters which determine the differentiation probability, such as the average generation time of stem cells $\tau_{c}$ (Figure 2(a)). Furthermore, it is predicted that the process of clone exhaustion can be accelerated by system perturbations, for example, due to repeated cell kill events (Figure 2(b)).

There is another point that might considerably affect the interpretation of experimental observations on clonal contribution. This is the fact that clone sizes (i.e., cell numbers per clone) are predicted to fluctuate over time. Therefore, also clones that actually contribute to hematopoiesis might be overlooked, for example, due to a threshold-dependent detection procedure. To illustrate this effect, consider the model results shown in Figure 3. Figure 3(a) illustrates the fluctuating size of 50 individual clones within a homeostatic system. In contrast, Figures $3(\mathrm{~b})$ and $3(\mathrm{c})$ are depicting different projections of this data. Whereas Figure 3(b) shows all existing clones (i.e., clone sizes larger than or equal to one cell), Figure 3(c) indicates measurable clones, assuming a detection threshold of 10 stem cells per clone. The emerging pattern looks very different although the underlying system is identical.

Applying these simulation results to different observations can help to identify misleading aspects in the interpretation of experimental findings and to disentangle seemingly contradictory results. One example is the ongoing debate, whether hematopoiesis is mono-, oligo- or polyclonal in nature. Opposing results, reaching from oligoclonality with large long-lived clones to polyclonal situations with many short-lived clones, have been reported [37-43]. To discuss the model analysis of these phenomena, let us 


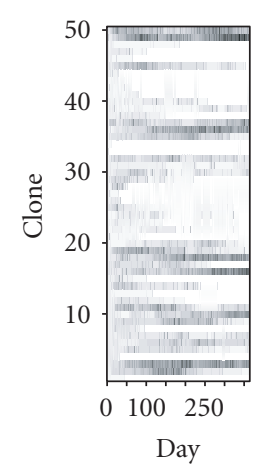

(a)

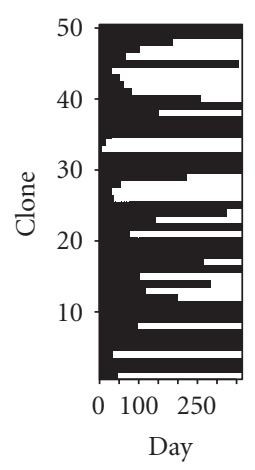

(b)

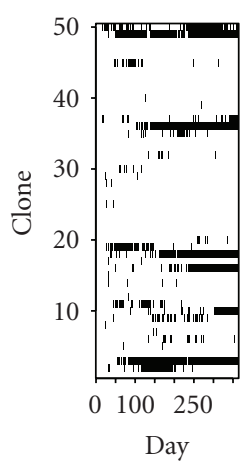

(c)

FIGURE 3: Detectability of individual clones. Simulated one-year followup of stem cell clones in a homeostatic reference system with 50 individually labeled stem cells randomly chosen at time 0 . Each horizontal bar represents one clone. (a) Real clone size with brightness indicating the contained cell number (light gray: low cell numbers; black: high cell numbers). (b) Existence of these clones (black), that is, all clones containing at least one cell are shown. (c) Detectable clones (black) using a detection threshold of at least 10 cells.

consider two particular results on the clonal composition of the hematopoietic system. Whereas Jordan and Lemischka observed an oligoclonal hematopoiesis with a few dominant persistent clones [37], Drize et al. reported a polyclonal composition with many small short-lived clones [39]. Although a similar general experimental setup for the tracing of retrovirally marked clones had been applied in both studies, the sampling strategies as well as the measurement protocols differed. In contrast to Jordan \& Lemischka who analyzed repeated blood/spleen samples with a high cell number but with relatively low detection sensitivity for individual marker signals, Drize et al. analyzed single-cell-induced spleen colonies obtained by injecting repeated bone marrow samples into irradiated recipient mice. Because only a small proportion of bone marrow cells seed in the spleen, the sample size of analyzed cells is small. However, this procedure ensures a high detection sensitivity due to the amplification of the marker signal in the clonally derived colonies.

To simulate these two experimental strategies, the following assumptions have been made. Model systems are initiated with individually labeled stem cells sampled from a homeostatic reference system. According to the two described experimental protocols, different numbers of marked cells, with $n=10$ cells for the Jordan-like simulation and $n=100$ cells for the simulation of the Drize experiment, were used. Experimentally detectable clones have been simulated by the sampling of individual model stem cells (representing spleen-colony forming cells) with a probability of 0.01 for the Drize-like setting, and by counting all differentiated clones (representing the entirety of bone marrow/spleen cells) which exceed a size threshold of 10000 cells per clone for the Jordan-like setting. This procedure is applied at sequential time points (3-month intervals). As demonstrated by our simulations (Figure 4), the different experimental ob-

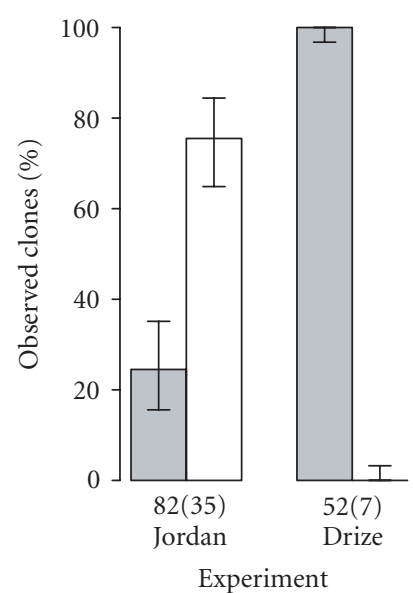

(a)

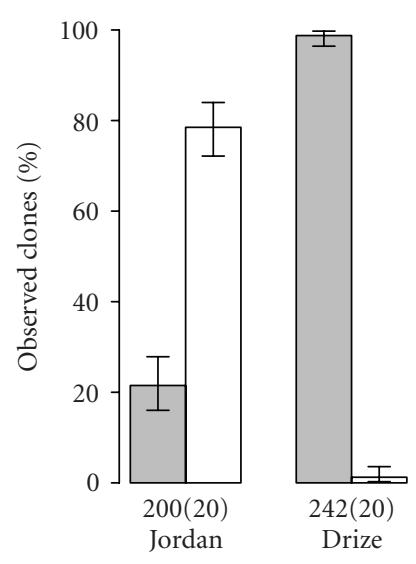

Simulation

(b)
FIGURE 4: Individual clone tracking results. Bars show proportions (mean, 95\% confidence interval) of individually marked clones. Shaded bars show short-lived clones (observed three months or less); empty bars show long-lived clones (observed more than three months). The number of analyzed clones (the number of mice/simulations runs) is given below the bars. (a) Experimental results taken from $[37,39]$; (b) respective simulation results, obtained by an identical underlying system, but applying different sampling and measuring strategies according to the experimental protocols.

servations can be consistently explained by differences in the sampling techniques and detection thresholds applied to an identical underlying biological system.

\section{ASYMMETRY OF CELLULAR FATE}

Although our model of a self-organized stem cell population does explicitly preclude asymmetric cell divisions, it still accounts for asymmetric cell fates. This asymmetry, however, is not caused by a predefined cell intrinsic program, but emerges as the result of cell-cell and cell-microenvironment interactions. For illustration (cf. Figure 5), consider a model cell with initial affinity $a_{1}$. Whenever this cell divides, it generates two identical daughter cells. However, during completion of a cell division, also the affinity $a$ changes from $a_{1}$ to a new value $a_{2}<a_{1}$. Now, one daughter cell might change to GE-A, subsequently regaining the affinity to its initial value $a_{1}$, while the other daughter cell continues to decrease $a$. Beside such an asymmetric development, also two scenarios of symmetric cell fates can be obtained: whenever both daughter cells regenerate their affinity, the number of cells with the original functional potential is amplified. In contrast, a symmetric differentiation is generated if both daughter cells remain under the influence of GE- $\Omega$.

It is also possible to quantitatively describe experimental data on asymmetric stem cell behavior within the context of our model. As an example, consider the cycling activity of stem cells, as described by Punzel et al. [44]. These authors analyzed the in vitro cell cycle activity of purified human cord blood cells. In short, individual CD $34^{+} / \mathrm{CD} 38^{-}$ 

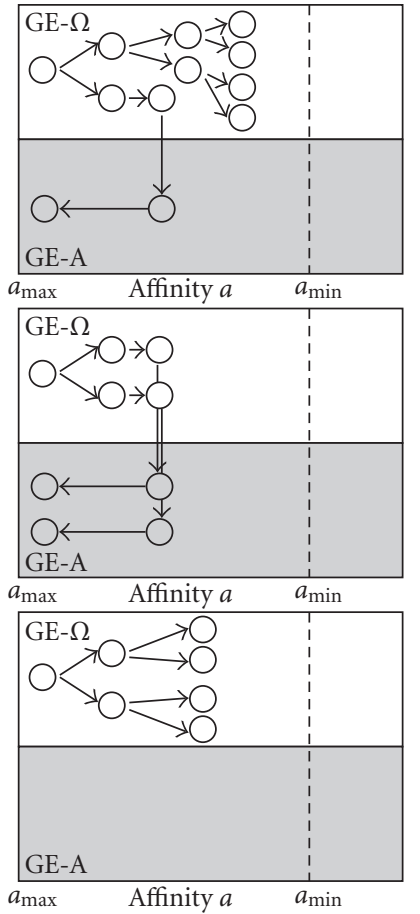

(a)
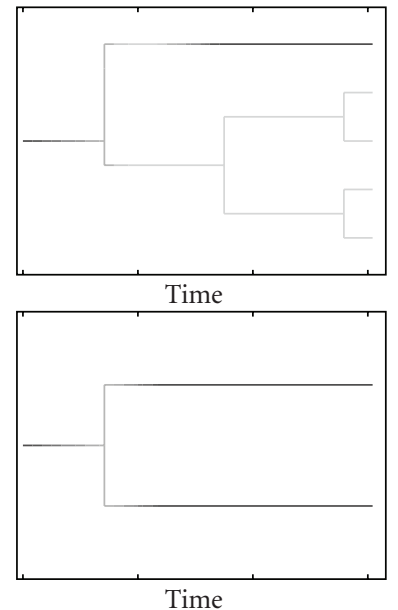

Time

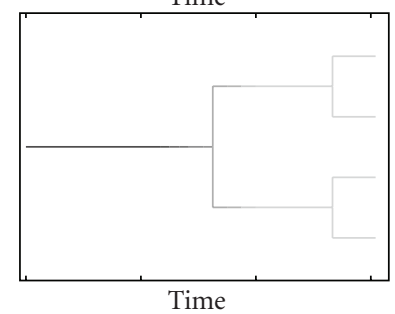

(b)
FIgURE 5: Self-renewing and differentiating stem cell fates. The schemes illustrate the realization of asymmetric (self-maintaining), symmetric self-renewing, and symmetric differentiating stem cell fates in the context of the self-organizing stem cell model. (a) Clonal development with respect to the two model growth environments and the affinity $a$. (b) Corresponding clone tree representations (cell fate over time) with gray-scale coding of actual affinities (dark gray: high $a$, light gray: low $a$ ).

cells were seeded into 96-well plates, previously coated with either bovine serum albumin (BSA), fibronectin (FN), or a specific stromal cell line feeder layer (AFT024). Using timelapse fluorescence microscopy, the division fate of each cell was traced over 10 days. A division is denoted as asymmetric if one first-generation daughter cell did not divide during the culture period while the other first-generation daughter divided at least once. Occurrence of asymmetric cell division was quantified by the percentage of cells showing asymmetric division with respect to all cells deposited ( $\mathrm{AD}$ index). The determined $\mathrm{AD}$ values for the stroma-free cultures (BSA, FN) were $22.9 \%$ and $22.8 \%$, respectively. In contrast, an $\mathrm{AD}$ value of $31.1 \%$ has been observed for the AFT024 cultures, suggesting that stromal coculture is able to increase the asymmetric behavior.

To test whether these results can quantitatively be reproduced without the assumption of asymmetric cell division events, individual model systems have been initiated with single cells. These systems have been traced for 10 days according to the experimental protocol. To compare the $\mathrm{AD}$ index of simulations and experiments, a model division is denoted as asymmetric whenever only one of the two firstgeneration daughter cells is performing further cell divisions. Otherwise, the division is denoted as symmetric.

The simulations revealed that the proportion of asymmetric cell fate is particularly sensitive to the initial affinity $a$ of in silico culture-initiating cells. The higher the initial GE-A affinity $a$ of the cells is, the higher the proportion of asymmetric cell divisions is (Figure 6). Because affinity $a$ directly correlates to the probability of a cell to long-term repopulate a model system, these results predict that cells with high long-term repopulating potential more frequently exhibit an asymmetric cell fate in vitro. Furthermore, the experimental observation that stromal coculture of stem cells enhances the proportion of asymmetric divisions can be explained by the assumption of different regeneration coefficients $r$. Whereas $r=1$ (i.e., no regeneration of affinity $a$ in GE-A) produces lower proportions of asymmetric cell fates (Figure 6(a)), $r>1$ leads to an increase in the amount of asymmetric cell fates (Figure 6(b)). Therefore, the heterogeneity of the in vitro stem cell supporting potential of different stromal cell types can consistently be represented in the model by growth environments (GE-A) allowing for variable degrees of $a$-regeneration.

Based on these results, we are able to quantitatively reproduce the published experimental results. Again starting from the previously derived reference parameter set that consistently describes different in vivo assays using C57BL/6 mice [35], a variation of the initial affinity range and of the regeneration coefficient lead to a good quantitative fit of simulation results and experimental data (Figure 7). Whereas the stroma-free situation is described by a regeneration coefficient of $r=1, r=1.05$ is assumed for the situation of a stroma-supported culture. Note that the latter $r$-value is still smaller than 1.1, which is the regeneration coefficient assumed for the in vivo situation. Both simulation scenarios use initial affinities $a$, uniformly distributed on the interval $[0.5 ; 1]$.

\section{CONCLUSIONS}

Particularly with regard to stem cell fate and individual clonal dynamics, there are a number of predictions arising from the proposed mathematical model. One basic conclusion is that the developmental fate of a stem cell cannot be predicted with certainty, even if the actual state of the cell could be determined exactly. However, probabilistic statements about the future development of individual clones as well as about the potential of a population of well-characterized cells are certainly possible. In terms of the model, the likelihood for a certain developmental fate of a stem cell is assumed to depend on the general potential of the cell, on its actual state, and on the microenvironmental signals the cell receives. As demonstrated for a chimeric mouse model [35], genetic differences in the potential of cells (e.g., reactivity on microenvironmental signals) are able to induce competitive growth (dis)advantages. It has be shown that even very small differences in cellular properties, which would not affect the general repopulation ability of the cells in a nonchimeric 


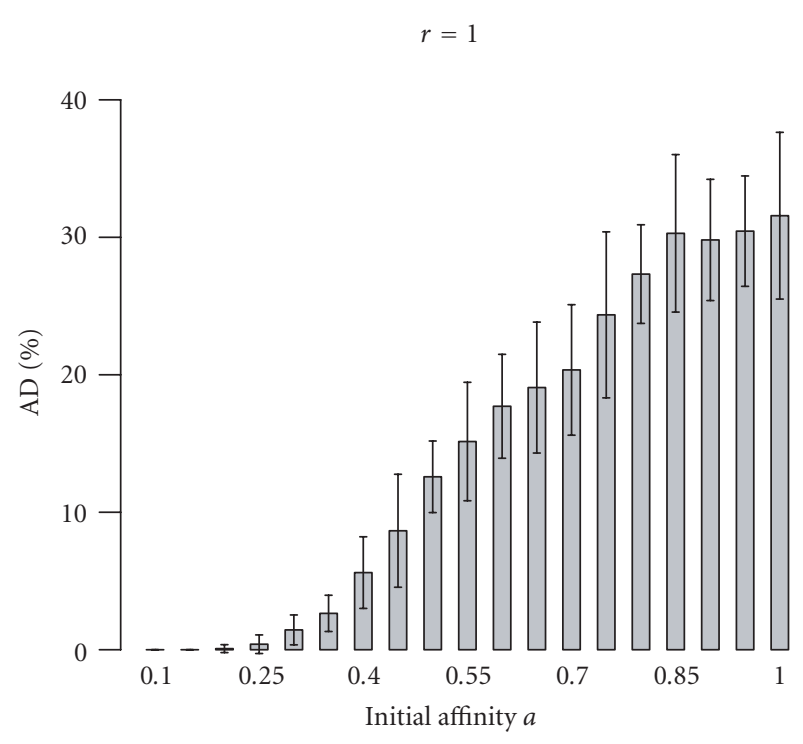

(a)

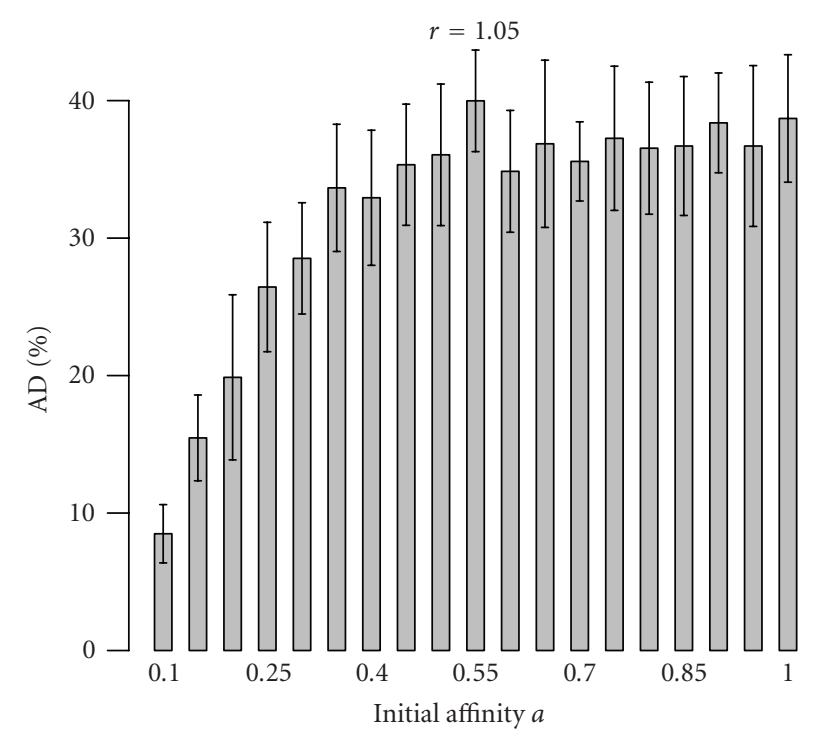

(b)

FIGURE 6: Heterogeneity of asymmetric stem cell fates. The proportions of asymmetric divisions (AD score; mean $+/-$ standard deviation) depending on the state of the culture initiating cell with respect to affintiy $a$ is shown: (a) nonregenerating situation (regeneration coefficient $r=1)$; (b) regenerating situation $(r=1.05)$.

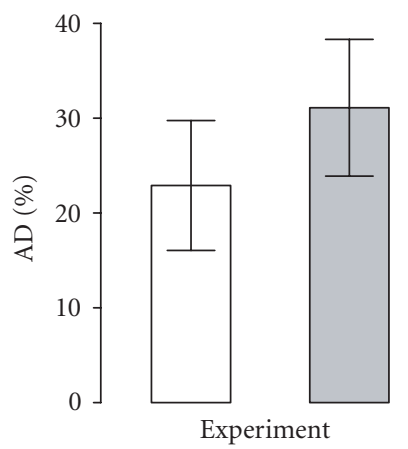

(a)

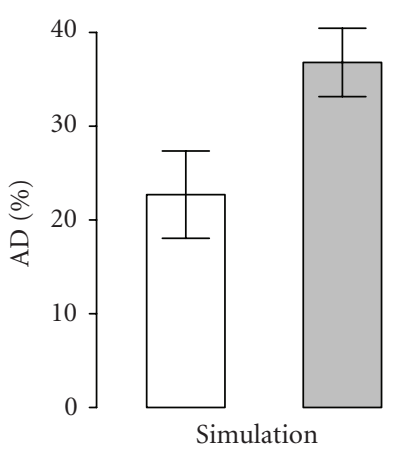

(b)
FIgURE 7: In vitro cell fates with respect to cell cycle activity. Bars represent the proportions of asymmetric divisions (AD score; mean $+/$ - standard deviation) in cultures with or without stromal support. Experimental results (taken from [44]) are based on $n=13$ independent evaluations of 96-well plates for culture conditions with (gray) and without stromal support (white). Corresponding simulation results have been obtained by evaluating $n=100$ in silico experiments per setting, each consisting of 96 individual, singlecell-induced model systems with regeneration coefficients $r=1.05$ (gray) and $r=1$ (white), respectively.

situation, are sufficient to sensitively affect the cellular development in the competition scenario. This might not only hold for cells of different genetic backgrounds. Also epigenetically determined (as, e.g., suggested by the group of MüllerSieburg $[45,46]$ ) or induced (e.g., by insertional mutagenesis
$[47,48])$ differences between stem cell clones within one genetic background could influence the probabilities for certain developmental fates.

Another related prediction is the clonality conversion as a consequence of system immanent fluctuations. Even in the oversimplified case of an identical potential of all stem cell clones, the dominance of some clones in the long run is predicted with certainty. Still, it is not possible to unequivocally specify the successful clones in advance. However, as stated above, even small differences in the cellular potential of stem cell clones are able to bias the competitive potential considerably. Our model is able to estimate the effect of differences in cellular parameters on competitive growth characteristics, and therefore, to provide statistical predictions about future clonal contributions. This might particularly be important to understand the effects of insertional mutagenesis as well as to quantitatively characterize the outcome of gene-therapeutic interventions.

A third important model prediction touches the role of asymmetric stem cell fates. Even though a developmental asymmetry of stem cells is inevitably required to provide a continuous production of differentiated cells without exhausting the stem cell population, this asymmetry is not necessarily linked to cell division events. Alternatively a flexible functional asymmetry can be achieved by a self-organizing population of interacting cells, including a certain degree of reversibility in cellular properties and functionalities.

Summarizing our results, we demonstrated that it is possible to understand tissue stem cell systems without assumptions on unidirectional developmental hierarchies, preprogrammed asymmetric division events, or other assumptions 
implying the existence of a predetermined stem cell entity. As illustrated for the hematopoietic system, a self-organizing perspective would change the paradigm of thinking about stem cells. Within such a concept, cellular properties are considered to permanently fluctuate with some cells meeting a situation of clonal expansion. This means that stem cells are selected and modified in response to cell-cell and cellmicroenvironment interactions, rather than being specialized a priori. Thus, it is their potential and the flexibility to use it, but not a particular actually expressed property, that distinguishes them from other cells.

\section{ACKNOWLEDGMENT}

This work has been supported by the German Research Foundation (DFG), Grant RO3500/1-1.

\section{REFERENCES}

[1] C. S. Potten and M. Loeffler, "Stem cells: attributes, cycles, spirals, pitfalls and uncertainties. Lessons for and from the crypt," Development, vol. 110, no. 4, pp. 1001-1020, 1990.

[2] D. Metcalf, "Hematopoietic regulators: redundancy or subtlety?" Blood, vol. 82, no. 12, pp. 3515-3523, 1993.

[3] G. de Haan, B. Dontje, C. Engel, M. Loeffler, and W. Nijhof, "The kinetics of murine hematopoietic stem cells in vivo in response to prolonged increased mature blood cell production induced by granulocyte colony-stimulating factor," Blood, vol. 86, no. 8, pp. 2986-2992, 1995.

[4] I. Roeder, G. de Haan, C. Engel, W. Nijhof, B. Dontje, and M. Loeffler, "Interactions of erythropoietin, granulocyte colonystimulating factor, stem cell factor, and interleukin-11 on murine during simultaneous administration," Blood, vol. 91, no. 9, pp. 3222-3229, 1998.

[5] R. Schofield, "The relationship between the spleen colonyforming cell and the haemopoietic stem cell. A hypothesis," Blood Cells, vol. 4, no. 1-2, pp. 7-25, 1978.

[6] I. R. Lemischka, "Microenvironmental regulation of hematopoietic stem cells," Stem Cells, vol. 15, supplement 1, pp. 63-68, 1997.

[7] F. M. Watt and B. L. M. Hogan, "Out of eden: stem cells and their niches," Science, vol. 287, no. 5457, pp. 1427-1430, 2000.

[8] P. J. Quesenberry, G. Colvin, and M. Abedi, "Perspective: fundamental and clinical concepts on stem cell homing and engraftment: a journey to niches and beyond," Experimental Hematology, vol. 33, no. 1, pp. 9-19, 2005.

[9] K. A. Moore and I. R. Lemischka, "Stem cells and their niches," Science, vol. 311, no. 5769, pp. 1880-1885, 2006.

[10] J. Zhang, C. Niu, L. Ye, et al., "Identification of the haematopoietic stem cell niche and control of the niche size," Nature, vol. 425, no. 6960, pp. 836-841, 2003.

[11] L. M. Calvi, G. B. Adams, K. W. Weibrecht, et al., "Osteoblastic cells regulate the haematopoietic stem cell niche," Nature, vol. 425, no. 6960, pp. 841-846, 2003.

[12] M. A. Kirkland, "A phase space model of hemopoiesis and the concept of stem cell renewal," Experimental Hematology, vol. 32, no. 6, pp. 511-519, 2004.

[13] M. Loeffler and I. Roeder, "Tissue stem cells: definition, plasticity, heterogeneity, self-organization and models - a conceptual approach," Cells Tissues Organs, vol. 171, no. 1, pp. 8-26, 2002.
[14] H. M. Blau, T. R. Brazelton, and J. M. Weimann, "The evolving concept of a stem cell: entity or function?” Cell, vol. 105, no. 7, pp. 829-841, 2001.

[15] N. D. Theise and I. Wilmut, "Cell plasticity: flexible arrangement," Nature, vol. 425, no. 6953, p. 21, 2003.

[16] S. J. Morrison and I. L. Weissman, "The long-term repopulating subset of hematopoietic stem cells is deterministic and isolatable by phenotype," Immunity, vol. 1, no. 8, pp. 661-673, 1994.

[17] N. Uchida, L. Jerabek, and I. L. Weissman, "Searching for hematopoietic stem cells. II. The heterogeneity of Thy1.1(lo) Lin(-/lo)Sca- $1^{+}$mouse hematopoietic stem cells separated by counterflow centrifugal elutriation," Experimental Hematology, vol. 24, no. 5, pp. 649-659, 1996.

[18] M. A. Goodell, K. Brose, G. Paradis, A. S. Conner, and R. C. Mulligan, "Isolation and functional properties of murine hematopoietic stem cells that are replicating in vivo," Journal of Experimental Medicine, vol. 183, no. 4, pp. 1797-1806, 1996.

[19] M. A. Goodell, S. McKinney-Freeman, and F. D. Camargo, "Isolation and characterization of side population cells," Methods in Molecular Biology, vol. 290, pp. 343-352, 2005.

[20] D. Surdez, B. Kunz, A. J. Wagers, I. L. Weissman, and A. V. Terskikh, "Simple and efficient isolation of hematopoietic stem cells from H2K-zFP transgenic mice," Stem Cells, vol. 23, no. 10, pp. 1617-1625, 2005.

[21] A. G. Rolink, S. L. Nutt, F. Melchers, and M. Busslinger, "Longterm in vivo reconstitution of T-cell development by Pax5deficient B-cell progenitors," Nature, vol. 401, no. 6753, pp. 603-606, 1999.

[22] T. Sato, J. H. Laver, and M. Ogawa, "Reversible expression of CD34 by murine hematopoietic stem cells," Blood, vol. 94, no. 8, pp. 2548-2554, 1999.

[23] P. Quesenberry, H. Habibian, M. Dooner, et al., "Physical and physiological plasticity of hematopoietic stem cells," Blood Cells, Molecules, and Diseases, vol. 27, no. 5, pp. 934-937, 2001.

[24] A. E. Frimberger, C. I. McAuliffe, K. A. Werme, et al., "The fleet feet of haematopoietic stem cells: rapid motility, interaction and proteopodia," British Journal of Haematology, vol. 112, no. 3, pp. 644-654, 2001.

[25] H. K. Habibian, S. O. Peters, C. C. Hsieh, et al., "The fluctuating phenotype of the lymphohematopoietic stem cell with cell cycle transit," Journal of Experimental Medicine, vol. 188, no. 2, pp. 393-398, 1998.

[26] G. B. Bradford, B. Williams, R. Rossi, and I. Bertoncello, "Quiescence, cycling, and turnover in the primitive hematopoietic stem cell compartment," Experimental Hematology, vol. 25, no. 5, pp. 445-453, 1997.

[27] S. H. Cheshier, S. J. Morrison, X. Liao, and I. L. Weissman, "In vivo proliferation and cell cycle kinetics of long-term selfrenewing hematopoietic stem cells," Proceedings of the $\mathrm{Na}$ tional Academy of Sciences of the United States of America, vol. 96, no. 6, pp. 3120-3125, 1999.

[28] M. A. Goodell, "CD34 ${ }^{+}$or $\mathrm{CD} 34^{-}$: does it really matter?" Blood, vol. 94, no. 8, pp. 2545-2547, 1999.

[29] C. R. R. Bjornson, R. L. Rietze, B. A. Reynolds, M. C. Magli, and A. L. Vescovi, "Turning brain into blood: a hematopoietic fate adopted by adult neural stem cells in vivo," Science, vol. 283, no. 5401, pp. 534-537, 1999.

[30] J. E. Grove, E. Bruscia, and D. S. Krause, "Plasticity of bone marrow-derived stem cells," Stem Cells, vol. 22, no. 4, pp. 487500, 2004.

[31] U. Lakshmipathy and C. Verfaillie, "Stem cell plasticity," Blood Reviews, vol. 19, no. 1, pp. 29-38, 2005. 
[32] N. D. Theise and R. Harris, "Postmodern biology: (adult) (stem) cells are plastic, stochastic, complex, and uncertain," Handbook of Experimental Pharmacology, no. 174, pp. 389408, 2006.

[33] I. Roeder and M. Loeffler, "A novel dynamic model of hematopoietic stem cell organization based on the concept of within-tissue plasticity," Experimental Hematology, vol. 30, no. 8, pp. 853-861, 2002.

[34] I. Roeder, M. Loeffler, P. J. Quesenberry, G. A. Colvin, and J.F. Lambert, "Quantitative tissue stem cell modeling," Blood, vol. 102, no. 3, pp. 1143-1145, 2003, author reply 1144-1145.

[35] I. Roeder, L. M. Kamminga, K. Braesel, B. Dontje, G. de Haan, and M. Loeffler, "Competitive clonal hematopoiesis in mouse chimeras explained by a stochastic model of stem cell organization," Blood, vol. 105, no. 2, pp. 609-616, 2005.

[36] I. Roeder, M. Horn, I. Glauche, A. Hochhaus, M. C. Mueller, and M. Loeffler, "Dynamic modeling of imatinib-treated chronic myeloid leukemia: functional insights and clinical implications," Nature Medicine, vol. 12, no. 10, pp. 1181-1184, 2006.

[37] C. T. Jordan and I. R. Lemischka, "Clonal and systemic analysis of long-term hematopoiesis in the mouse," Genes and Development, vol. 4, no. 2, pp. 220-232, 1990.

[38] B. Capel, R. G. Hawley, and B. Mintz, "Long- and short-lived murine hematopoietic stem cell clones individually identified with retroviral integration markers," Blood, vol. 75, no. 12, pp. 2267-2270, 1990.

[39] N. J. Drize, J. R. Keller, and J. L. Chertkov, "Local clonal analysis of the hematopoietic system shows that multiple small short-living clones maintain life-long hematopoiesis in reconstituted mice," Blood, vol. 88, no. 8, pp. 2927-2938, 1996.

[40] H. J. Kim, J. F. Tisdale, T. Wu, et al., "Many multipotential gene-marked progenitor or stem cell clones contribute to hematopoiesis in nonhuman primates," Blood, vol. 96, no. 1, pp. 1-8, 2000.

[41] Y. Guan, S. Ralph, and D. E. Hogge, "Polyclonal normal hematopoietic progenitors in patients with acute myeloid leukemia," Experimental Hematology, vol. 30, no. 7, pp. 721$728,2002$.

[42] F. Guidetti, S. Grazioli, F. Capelli, et al., "Primitive hematopoietic stem cells show a polyclonal pattern in myelodysplastic syndromes," Haematologica, vol. 89, no. 1, pp. 21-28, 2004.

[43] H. Glimm, M. Schmidt, M. Fischer, et al., "Efficient marking of human cells with rapid but transient repopulating activity in autografted recipients," Blood, vol. 106, no. 3, pp. 893-898, 2005.

[44] M. Punzel, D. Liu, T. Zhang, V. Eckstein, K. Miesala, and A. D. Ho, "The symmetry of initial divisions of human hematopoietic progenitors is altered only by the cellular microenvironment," Experimental Hematology, vol. 31, no. 4, pp. 339-347, 2003.

[45] C. E. Müller-Sieburg, R. H. Cho, M. Thoman, B. Adkins, and H. B. Sieburg, "Deterministic regulation of hematopoietic stem cell self-renewal and differentiation," Blood, vol. 100, no. 4, pp. 1302-1309, 2002.

[46] C. E. Müller-Sieburg and H. B. Sieburg, "Clonal diversity of the stem cell compartment," Current Opinion in Hematology, vol. 13, no. 4, pp. 243-248, 2006.

[47] C. Baum, C. von Kalle, F. J. T. Staal, et al., "Chance or necessity? Insertional mutagenesis in gene therapy and its consequences," Molecular Therapy, vol. 9, no. 1, pp. 5-13, 2004.

[48] O. Kustikova, B. Fehse, U. Modlich, et al., "Clonal dominance of hematopoietic stem cells triggered by retroviral gene marking," Science, vol. 308, no. 5725, pp. 1171-1174, 2005. 

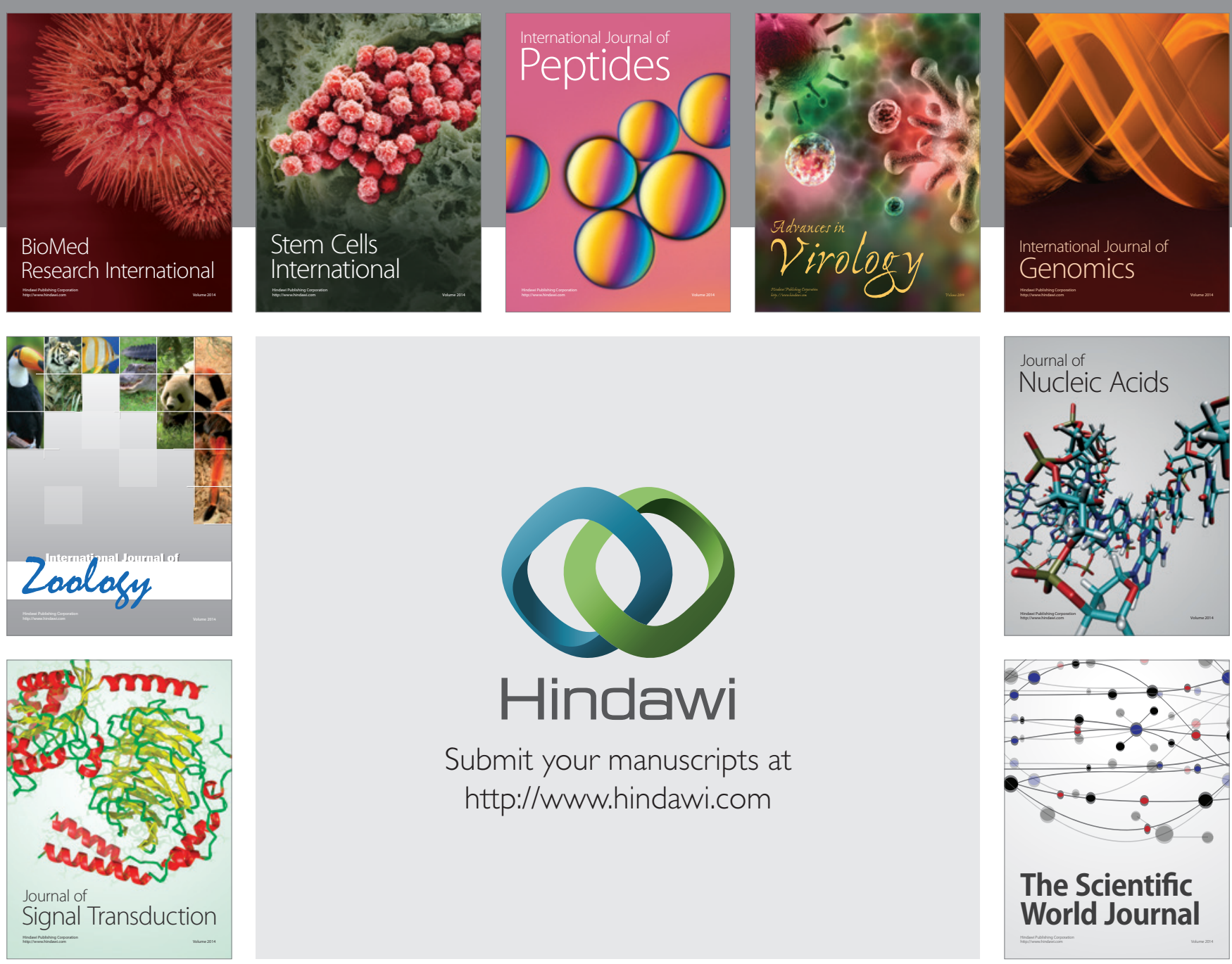

Submit your manuscripts at

http://www.hindawi.com
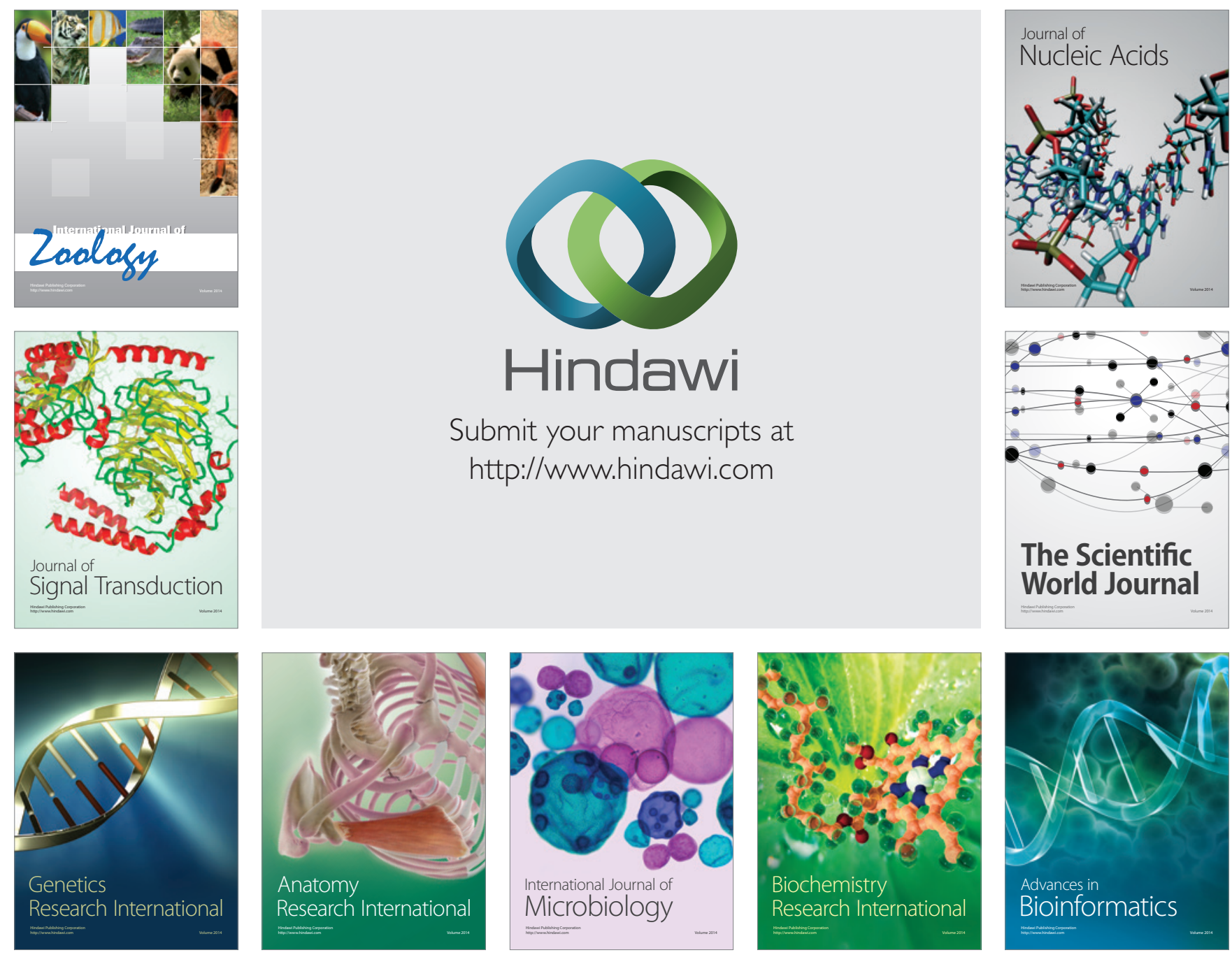

The Scientific World Journal
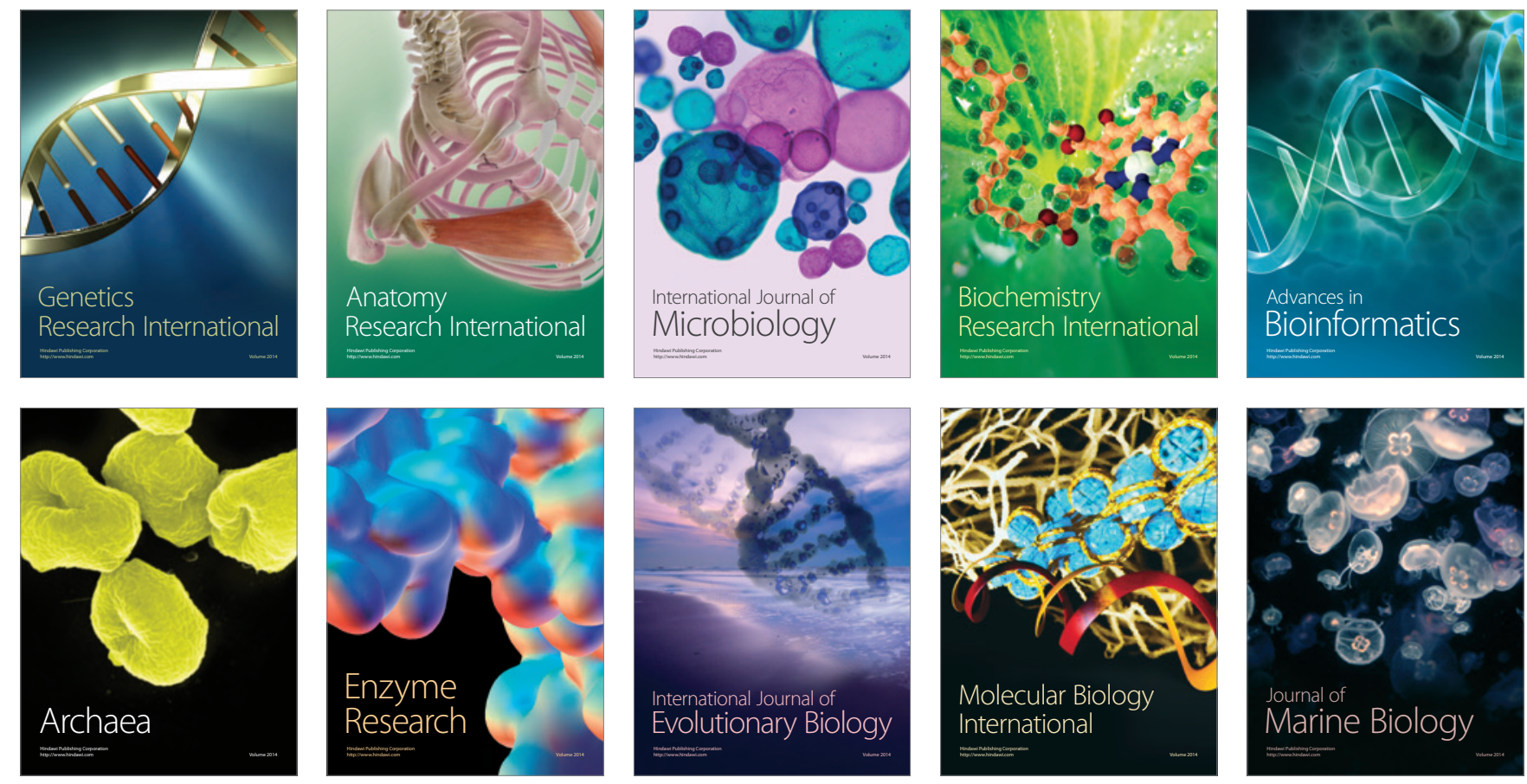